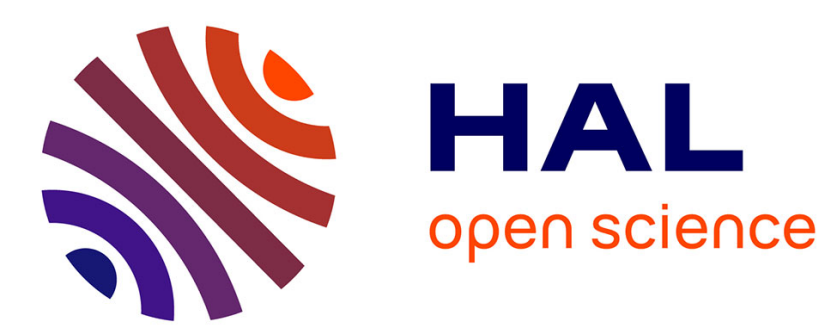

\title{
POLARISATION EFFECTS IN ENERGY-TRANSFER COLLISIONS BETWEEN LASER-EXCITED ATOMS
}

\author{
G. Nienhuis
}

\section{To cite this version:}

G. Nienhuis. POLARISATION EFFECTS IN ENERGY-TRANSFER COLLISIONS BETWEEN LASER-EXCITED ATOMS. Journal de Physique Colloques, 1985, 46 (C1), pp.C1-97-C1-106. 10.1051/jphyscol:1985109 . jpa-00224479

\section{HAL Id: jpa-00224479 https://hal.science/jpa-00224479}

Submitted on 1 Jan 1985

HAL is a multi-disciplinary open access archive for the deposit and dissemination of scientific research documents, whether they are published or not. The documents may come from teaching and research institutions in France or abroad, or from public or private research centers.
L'archive ouverte pluridisciplinaire HAL, est destinée au dépôt et à la diffusion de documents scientifiques de niveau recherche, publiés ou non, émanant des établissements d'enseignement et de recherche français ou étrangers, des laboratoires publics ou privés. 
POLARISATION EFFECTS IN ENERGY-TRANSFER COLLISIONS BETWEEN LASEREXCITED ATOMS

\author{
G. Nienhuis \\ Eysisch Laboratorium, Rijksuniversiteit Utrecht, Postbus 80 000, \\ 3508 TA Utrecht, The Netheriands
}

Résumé - Nous étudions 1'équation pilote gênêralisẻe pour la matrice densité d'un atome à deux niveaux dégénérés dans un champ laser ayant une polarisation arbitraire. Cette étude est utilisée pour 7 'analyse des collisions de transfert d'énergie entre deux atomes excités par laser, en termes des taux collisionnels pour les sous-états magnétiques. Les résultats sont appliquës à nos récentes mesures de l'ionisation assocjative entre deux atomes $\mathrm{Na}(3 \mathrm{P})$ excités et polarisés par laser.

Abstract - The analysis of energy-transfer collisions between laser-excited atoms in terms of rate constants for separate magnetic substates requires knowledge of the excited-state density matrix for each applied laser polarisation. We study the generalised rate equation for this density matrix. Then we describe the relation between the collision signals and the M-dependent collision amplitudes. The results are applied to our recent measurements of associative ionisation of laser-excited $\mathrm{Na}(3 \mathrm{P})$ atoms.

\title{
I - OBSERVABILITY OF POLARISATION EFFECTS
}

Polarisation effects in inelastic atomic collisions can be studied by two distinct methods, which are basically each other's time reverse. One may analyse the polarisation properties or the angular distribution of the radiation emitted by one or both of the atoms in this final excited state. Alternatively, one may prepare one or both of the atoms in the excited state by laser excitation prior to the collision. Both techniques give information on partial cross sections for transitions to or from the magnetic substates $\left|M_{e}\right\rangle$ of the excited state. The similarity and the difference between these two methods is aptly il7ustrated by considering the information content in terms of the scattering amplitudes $f\left(M_{e}\right)$. For notational convenience we "first" consider the case that only one of the collision partners takes part in the radiation process.

When $f\left(M_{e}\right)$ is the scattering amplitude for excitation of the magnetic substate $\mid M_{e}>$, then the excited state emerging from the collision is described by the density matrix $\rho$, with matrix elements.

$$
\left.<M_{e}|\rho| M_{e}{ }^{\prime}\right\rangle=\int d \Omega\left[f\left(M_{e}\right) \cdot f^{*}\left(M_{e}{ }^{\prime}\right)\right]_{a v},
$$

where the average is over the initial relative velocity and the substates of the (usually unpolarised) initial state, and an integration is performed over the direction $\Omega$ of the relative velocity after the collision. When one of the scattered atoms is detected in coincidence with the photon emitted after the collision, this integration extends over the angles selected by the aperture of the detector.

The intensity of the radiation with polarisation $\vec{\varepsilon}$ emitted by spontaneous decay from the excited state to a lower state (usually the ground state) with magnetic 
substates $\left|M_{\mathrm{g}}\right\rangle$ is determined by the density matrix $\rho$, according to the proportionality relation $/ 1 /$

$$
\left.I(\varepsilon) \propto \sum_{M_{g}} M_{e} M_{e}{ }^{\prime}<M_{g}\left|d^{+}\right| M_{e}\right\rangle\left\langle M_{e}|\rho| M_{e}{ }^{\prime}>\left\langle M_{e}{ }^{\prime}|d| M_{g}\right\rangle=\operatorname{Tr} \rho D(\vec{\varepsilon}),\right.
$$

where

$$
\left.<M_{e}|d| M_{g}\right\rangle=\vec{\varepsilon} \cdot<M_{e}|\vec{\mu}| M_{g}>
$$

is a matrix element of the component of the atomic electric dipole in the polarisation direction, and where

$$
D(\vec{\varepsilon})=d d^{\dagger}
$$

serves as a detection operator. Hence the polarisation direction $\vec{\varepsilon}$, which is selected by the polarisation analyser in front of the photodetector fully determines the detection operator, which acts as a sieve that determines the piece of information on $\rho$ gained in the measurement. It is the density matrix $\rho$ that contains the information on the scattering process. In the ideal situation the polarisation analysis and the angular distribution of the photons determine $\rho$ completely. In general this is only true when the angular momentum $J_{e}$ of the excited state is not larger than 1 , since D contains only the multipolar ranks 0,1 and 2 when expanded in spherical tensors /2/. This results from the fact that $D$ is a simple product of the vector operators $\mathrm{d}$ and $\mathrm{d} \dagger$.

Very much the same information can be gained by studying the time reversed process, where the excited state is prepared by laser excitation as an initial state to the collision. The corresponding scattering amplitudes are denoted as $g\left(M_{e}\right)$, and they are linked by time reversal symmetry to $f\left(M_{e}\right)$, provided of course that the initial state in the first process is the same as the final state in the second process. When $\sigma$ is the density matrix of the initial state, as prepared by laser excitation, then the production rate $R$ of the final state obeys the proportionality relation.

$$
R \propto \sum_{f} f d \Omega\left[g\left(M_{e}\right)<M_{e} \int \sigma \mid M_{e}{ }^{\prime}>g^{*}\left(M_{e}^{\prime}\right)\right]_{a v}=\operatorname{Tr} \sigma_{e} G
$$

where $G$ is the detection operator with matrix elements

$$
\left\langle M_{e}{ }^{\prime}|G| M_{e}\right\rangle=\sum_{f} f d \Omega\left[g^{*}\left(M_{e}{ }^{\prime}\right) g\left(M_{e}\right]_{a v} .\right.
$$

The average in (1.5) and (1.6) is performed over the initial distribution of relative velocities. An integration is performed over the direction $\Omega$ of the relative velocities after the collision, and the summation is over the substates of the final state. In this case it is the detection operator $G$ that contains the information on the scattering process, and the characteristics of the exciting laser determine the initial density matrix $\sigma$, and thereby the piece of information on $G$ that is gained. A major advantage of this method of saturated laser excitation over the polarisation analysis of spontaneous emission is that the multipole rank $k$ of the initial density matrix $\sigma$ is not restricted to the values 0,1 or 2 . By a proper choice of the laser polarisation directions in successive measurements a full determination of $G$ is generally feasible.

The formal analogy between polarisation analysis of collisionally excited spontaneous emission and the time reverse orocess with a laser-excited initial state has been 
elaborated for electron-atom scattering /3/. The analogy holds equally well for collision processes in which two atoms are excited to an emitting excited state in a single collision /4/ as compared to collisions between two initially laser-excited atoms $/ 5 \%$. In the present paper we discuss the description of inelastic collisions between two laser-excited atoms, as a function of the polarisation of the laser. We study the validity of generalised rate equations in terms of Einstein coefficients as compared with Bloch-type equations in determining the stationary density matrix o of each one of the atoms. The description is applied to our current experiments on collisions between two laser-excited $\mathrm{Na}$ atoms, both in a single atomic beam and in two counterrunning beams, leading to associative ionisation. The reTation with other inelastic processes, such as energy pooling, is briefly discussed.

\section{II - BLOCH EQUATIONS AND GENERALISED RATE EQUATIONS}

An essential ingredient in the polarisation analysis of inelastic collisions with Taser-excited initial states is the specification of the stationary density matrix $\sigma_{e}$ of this excited state. This density matrix is a part of the combined density matrix $\sigma$ of the excited state and the ground state, which are coupled by the radiation field. The laser field is described as a classical oscilating field with a frequency distribution centered around $\omega_{L}$. In the rotating-wave approximation the atom-field coupling is given by

$$
H_{a f}(t)=-d E_{+}(t) e^{-i \omega L t}-d^{\dagger} E_{-}(t) e^{i \omega_{L} t}
$$

with defined as in (1.3) in terms of the polarisation $\vec{\varepsilon}$ of the exciting radiation. The time dependence of $E_{+}$and $E_{-}$allows for amplitude and frequency modulation, and thereby a finite laser bandwidth. We introduce the dimensionless operators $Q_{\sigma}$ by specifying their matrix elements as a Clebsch-Gordan coefficient, according to

$$
\left\langle M_{e}\left|Q_{\sigma}\right| M_{g}\right\rangle=\left\langle F_{e} M_{e} \mid F_{g} M_{g} ; 1 \sigma\right\rangle
$$

for $\sigma=-1,0,1$, with $F_{0}$ and $F_{g}$ the angular momenta of the excited state and the ground state. In a rotating frame, the Liouvilie equation for the complete density matrix o takes the familiar form of generalised Bloch equations $/ 6 /$, which read

$$
\begin{aligned}
& \frac{d}{d t} \sigma_{e}(t)=-A \sigma_{e}(t)+\frac{i}{\hbar}\left(E_{+}(t) d \sigma_{g e}(t)-\sigma_{e g}(t) d^{\dagger} E_{-}(t)\right) \\
& \frac{d}{d t} \sigma_{g}(t)=A \sum_{\sigma} Q_{\sigma}^{\dagger} \sigma_{e}(t) Q_{\sigma}+\frac{i}{\hbar}\left(E_{-}(t) d^{\dagger} \sigma_{e g}(t)-\dot{\sigma}_{g e}(t) d E_{+}(t)\right) \\
& \frac{d}{d t} \sigma_{e g}(t)=\left[i\left(\omega_{L}-\omega_{0}\right)-\frac{1}{2} A\right] \sigma_{e g}(t)+\frac{i}{\hbar}\left(E_{+}(t) d \sigma_{g}(t)-\sigma_{e}(t) d E_{+}(t)\right) \\
& \frac{d}{d t} \sigma_{g e}(t)=\left[-i\left(\omega_{L}-\omega_{0}\right)-\frac{1}{2} A\right] \sigma_{g e}+\frac{i}{\hbar}\left(E_{-}(t) d^{\dagger} \sigma_{e}(t)-\sigma_{g}(t) d^{\dagger} E_{-}(t)\right) .
\end{aligned}
$$

Here $\omega_{0}$ is the transition frequency between the states e and $g$. Spontaneous emission is measured by the Einstein coefficient $A$, and gives a gain term in the equation for 
$\sigma_{f}(t)$, and a loss term in the other equations. In monochromatic radiation, the positive- and negative-frequency parts $E_{+}$and $E_{-}$of the field are constants.

The Bloch equations (2.3) can be used to derive generalised rate equations for the populations of the excited state and the ground state by eliminating oeg and $\sigma_{g e}$, which is possible after some simplifying assumptions that are valid in special cases $/ 7 /$. The equations (2.3) for $\sigma_{e g}$ and $\sigma_{g e}$ can be formally written as an integral over $\sigma_{e}$ and $\sigma_{g}$ in the past, and we find

$$
\begin{aligned}
\sigma_{e g}(t)= & \int_{\infty}^{t} d t^{\prime} \exp \left[\left(i\left(\omega_{L}-\omega_{0}\right)-\right.\right. \\
& \left.\left.\frac{1}{2} A\right)\left(t-t^{\prime}\right)\right] \\
& \frac{j}{h}\left[E_{+}\left(t^{\prime}\right) d \sigma_{g}\left(t^{\prime}\right)-\sigma_{e}\left(t^{\prime}\right) d E_{+}\left(t^{\prime}\right)\right]
\end{aligned}
$$

and an analogous equation for $\sigma_{\text {ge }}$. Substituting these equations in the Bloch equations for $\sigma_{e}$ and $\sigma_{g}$ yields rate equations of the Master type for $\sigma_{e}$ and $\sigma_{g}$, provided that we may replace $\sigma_{e}\left(t^{\prime}\right)$ and $\sigma_{g}\left(t^{\prime}\right)$ by $\sigma_{e}(t)$ and $\sigma_{g}(t)$. This is justified only if $\sigma_{e}$ and $\sigma_{g}$ change negliglbly during the time of interest in the integrand. This time of interest is restricted by the spontaneous decay term $\frac{1}{2} A$, and possibly by the finite bandwidth of the laser radiation, which arises after substituting

$$
<E_{-}(t) E_{+}\left(t^{\prime}\right)>=\left(2 \varepsilon_{0} c\right)^{-1} \int d \omega \exp \left[i\left(\omega-\omega_{L}\right)\left(t-t^{\prime}\right)\right] I(\omega)
$$

for the average over the laser fluctuations, with $I(\omega)$ the spectral intensity of the laser. For a finite bandwidth $\lambda$, the relevant values of $t-t$ ' are restricted to at most $\lambda^{-1}$. When the stimulated-transition rate is smaller than the maximum of $A$ and $\lambda$, we can replace $\sigma_{e}\left(t^{\prime}\right)$ and $\sigma_{g}\left(t^{\prime}\right)$ by $\sigma_{e}(t)$ and $\sigma_{g}(t)$ in the integrand, and we obtain rate equations for $\sigma_{e}$ and $\sigma_{g}$, which do not contain $\sigma_{e g}$ and $\sigma_{g e}$. These rate equations are obtained in a dimensionless form if we introduce the real parameters $a$ and b by the definition

$$
a+i b=\frac{\pi^{2} c^{2}}{\hbar \omega_{0}^{3}} \int d \omega I(\omega)\left[\pi\left(\frac{1}{2} A+i\left(\omega-\omega_{0}\right)\right)\right]^{-1} .
$$

The factor in front of the integral is simply the ratio $B / A$ of the Einstein coefficient for stimulated emission and spontaneous emission, and the real part of the integral in $\{2.6\}$ is the overlap of the spectral intensity of the laser and the natural profile. Hence $a$ is the saturation parameter defined as the ratio of the stimulated to the spontaneous emission rate, and $b$ is a corresponding light-shift parameter. Furthermore we introduce the dimensiontess transition operator

$$
Q=\sum_{\sigma} Q_{\sigma} \vec{u}_{\sigma} \cdot \vec{\varepsilon}
$$

with $\vec{u}_{\sigma}$ the spherical unit vectors

$$
\vec{u}_{ \pm 1}=\frac{1}{2}(\mp \hat{x}-i \hat{y}) \quad, \vec{u}_{0}=\hat{z} .
$$

One notices that $Q$ becomes equal to $Q$ defined in (2.2) when the laser polarisation is equal to the spherical unit vector $\vec{u}_{\vec{u}}$. However the definition (2.7) allows for an arbitrary polarisation, that is not Recessarily circular or Tinear. Obviousiy, the dipole component $d$ is proportional to the operator, due to the Wigner-Eckart 
theorem $/ 2 /$.

The generalised equations for $\sigma_{e}$ and $\sigma_{g}$ take the form

$$
\begin{aligned}
\frac{1}{A} \frac{d}{d t} \sigma_{e}(t) & =-i 3 b\left[\frac{1}{2} Q Q^{\dagger}, \sigma_{e}(t)\right]-\sigma_{e}(t) \\
& +3 a\left(Q \sigma_{g}(t) Q^{\dagger}-\frac{1}{2} Q Q^{\dagger} \sigma_{e}(t)-\frac{1}{2} \sigma_{e}(t) Q Q^{\dagger}\right), \\
\frac{1}{A} \frac{d}{d t} \sigma_{g}(t) & =i 3 b\left[\frac{1}{2} Q^{\dagger} Q, \sigma_{g}(t)\right]+\sum_{\sigma} Q_{\sigma}^{\dagger} \sigma_{e}(t) Q \sigma \\
& +3 a\left(Q^{\dagger} \sigma_{e}(t) Q-\frac{1}{2} Q^{\dagger} Q \sigma_{g}(t)-\frac{1}{2} \sigma_{g}(t) Q Q\right)
\end{aligned}
$$

These equations are given in a dimensionless form, and they contain only multiplications with the operators $Q$ and $Q^{\dagger}$, which are defined in (2.2). For linear or circular polarisation, $Q$ is equal to one of the $Q$ when the quantisation axis is properly chosen. One immediately checks that in that case the density matrices $\sigma_{\mathrm{g}}$ and $\sigma_{e}$ are diagonal in the states $\left|M_{\mathrm{g}}\right\rangle$ and $\left|\mathrm{M}_{\mathrm{e}}\right\rangle$, provided that they were diagonal at some initial time. Hence in the case of linear or circular polarisation the state of the atom is entirely described in terms of populations of magnetic substates, and coherences do not occur. The Master equation for these populations is directly extracted from (2.9) in this case, and we find the usual equations $13 /$

$$
\begin{aligned}
\frac{1}{A} \dot{N}_{M_{e}} & =-N_{M_{e}}-3 a\left|<F_{e} M_{e}\right| F_{g} M_{e}-\sigma ; 1 \sigma>\left.\right|^{2}\left(N_{M_{e}}-n_{M_{e}}-\sigma\right) \\
\frac{1}{A} \dot{n}_{M_{g}} & =\sum_{M_{e}}\left|<F e^{M_{e}}\right| F_{g} M_{g} ; 1 M_{e}-M_{g}>\left.\right|^{2} N_{M_{e}} \\
& +3 a\left|<F e^{M_{g}}+\sigma\right| F_{g} M_{g} ; 1 \sigma>\left.\right|^{2}\left(N_{M_{g+\sigma}}-n_{M_{g}}\right)
\end{aligned}
$$

for $\vec{\varepsilon}=\vec{u}$, where $N$ denotes the populations of the excited substates, and $n$ the ground state. One notices that the first terms on the right-hand side of (2.9) do not contribute in this case, since the relevant operators are all diagonal and the commutators disappear.

In the case of elliptical polarisation it is not generally possible to choose a basis for which $\sigma_{e}$ and $\sigma_{g}$ are diagonal at all times, and we have to use the operator equations $(2.9)$, rather then the equations (2.10) for populations. This is an important remark, since, as we shall see, the use of elliptically polarised light can be necessary for a full determination of the detection operator $G$ in the case of collision experiments with laser-excited initial states. The commutators in (2.9) reflect energy shifts, which give rise to an effective Hamiltonian for the excited state and the ground state. The light-shift parameter $b$ vanishes when the laser spectrum is symmetrically distributed around the resonance frequency $\omega_{0}$. 
We demonstrated that the generalised rate equations (2.9) are valid when the stimulated-transition rates are small either compared with the natural decay rate or with the laser bandwidth. This is true when the saturation parameter a is small compared with 1 or with $\lambda$. In many cases of practical interest, where the atoms are excited by an intense narrow-band laser source, this condition is not fulfilled. Nevertheless, eqs. (2.9) can still be used to evaluate the stationary limit of the density matrices $\sigma_{e}$ and $\sigma_{g}$. This can be justified by inspecting eq. (2.4) and the analogous equation for $\sigma_{\mathrm{ge}}(t)$. In the stationary limit, the time dependence of $\sigma_{g}$ and $\sigma_{e}$ in the integrand of $(2.4)$ can be omitted. Substitution of the result in the first two equations (2.3) then leads to the same stationary values of $\sigma_{e}$ and $\sigma_{f}$ as the rate equations (2.9). Hence the Bloch equations (2.3), and the generalised rate equations (2.9) yield the same density matrices $\sigma_{e}$ and $\sigma_{g}$ in the stationary state, even though the time-dependent approach of the stationary state can be quite different according to these two sets of equations. This conclusion is of great importance to our present discussion, since the atoms usually have sufficient time to reach a steady state in the laser field before the beginning of the collision. This steady state is correctly predicted by eqs. (2.9) even for an intense monochromatic laser.

When the polarisation $\vec{\varepsilon}$ is equal to one of the spherical unit vectors $\vec{u}_{\sigma}$, i.e. for linear or circular polarisation, the steady state is diagonal, and the stationary populations are determined by eqs. (2.10) by setting the right-hand sides equal to zero. The stationary distribution of the excited atoms over the magnetic substates is independent of the intensity in this case, as has been shown before $/ 3,7 /$. When $F_{e}=F_{0}-1$, no stationary population of the excited state is possible when $\vec{\varepsilon}=\vec{u}_{\sigma}$. When $F_{e}=F_{g}$, a circular polarisation pumps all the atoms to the ground state with $M_{\mathrm{g}}= \pm F_{e}$ for $\sigma= \pm 1$. Linear polarisation $\left(\vec{\varepsilon}=\vec{u}_{\sigma}\right)$ leads to an isotropic stationary distribution over the excited substates when $F_{e}=F_{g}$ is a half integer, and no stationary excited atoms are possible when $F_{e}={ }^{g} F_{g}$ is integer /8/. Finally, when $F_{e}=F_{g}+1$, circular polarisation pumps the aloms to the states $M_{g}= \pm F_{g}, M_{e}= \pm F_{e}$ for $\sigma= \pm 1$. Linear polarisation produces a stationary excited distribution for $F_{e}=F_{g} \mp 1$ where $/ 3 /$

$$
N_{M}=n_{e}\left|<2 F_{e}-1,0\right| F_{g} M ; F_{e}-M>\left.\right|^{2}
$$

The total excitation fraction depends on the saturation parameter a, but the relative distribution is fully specified by the polarisation $\vec{\varepsilon}$ in these cases. The use of elliptical polarisation provides an additional possibility for modifying the excited state, as does the use of partially polarised radiation.

\section{III - POLARISATION-DEPENDENT RATE OF INELASTIC COLLISIONS}

We consider the case of inelastic collisions between two laser-excited atoms $A$ and $B$, which we shall take to be identical. The magnetic substates of the excited states are denoted as $\left|M_{A} M_{B}\right\rangle$. The production rate of final-state products can be expressed as in eq. (1.6), and we obtain the proportionality relation

$$
R \propto \operatorname{Tr} \sigma_{A} \cdot \sigma_{B} G
$$

with the detection operator 


$$
\left\langle M_{A} M_{B}|G| M_{A}{ }^{\prime} M_{B}{ }^{\prime}\right\rangle=\sum_{f} \int d \Omega\left[g\left(M_{A}{ }^{\prime} M_{B}{ }^{\prime}\right) g^{*}\left(M_{A} M_{B}\right)\right]_{a v} .
$$

We assume that the possible substates of the final state are not discriminated, and a summation over these substates is implied in (3.2). When both atoms are excited by the same laser beam, the two density matrices $\sigma_{A}$ and $\sigma_{B}$ are identical. Finally we consider the case that the direction of the scattered particles after the collision remains unobserved, so that the integration over the direction $\Omega$ of the final relative velocity extends over all directions. Then the symmetry properties of the detection operator G simply reflect the symmetry of the distribution of initial relative velocities. In the case of a single atomic beam or two counterrunning beams, $G$ has an axial symmetry. Then it obeys the symmetry relations

$$
\left\langle M_{A} M_{B}|G| M_{A}{ }^{\prime} M_{B}{ }^{\prime}\right\rangle=0 \text { for } M_{A}+M_{B} \neq M_{A}{ }^{\prime}+M_{B}{ }^{\prime} \text {, }
$$

where we assumed that the quantisation axis for the magnetic substates is taken along the beam axis. From reflection symmetry about a plane through this axis we obtain the relations

$$
\left\langle M_{A} M_{B}|G| M_{A}^{\prime} M_{B}^{\prime}\right\rangle=\left\langle-M_{A}-M_{B}|G|-M_{A}^{\prime}-M_{B}^{\prime}\right\rangle
$$

Since the colliding atoms are identical, $G$ obeys the relations

$$
\left\langle M_{A} M_{B}|G| M_{A}^{\prime} M_{B}^{\prime}\right\rangle=\left\langle M_{B} M_{A}|G| M_{B}^{\prime} M_{A}^{\prime}\right\rangle .
$$

Finally, the hermiticity of $G$ yields the equalities

$$
\left\langle M_{A} M_{B}|G| M_{A}^{\prime} M_{B}^{\prime}\right\rangle=\left\langle M_{A}^{\prime} M_{B}{ }^{\prime}|G| M_{A} M_{B}\right\rangle^{*}
$$

The collision process can be assumed to be unaffected by the state of the nuclear spins, and it is sufficient to take for $\sigma_{A}$ and $\sigma_{B}$ the partial trace over the nuclear spins. The magnetic quantum numbers $M_{A}$ and $M_{B}$ define the orientation of the electronic angular momentum $J$ of the atoms. Since the nuclear spin has sufficient time to couple to the electronic angular momentum $\mathrm{J}$ during the optical pumping, it is essential to describe the laser excitation in terms of the hyperfine levels. In fact, it is practical to tune the exciting laser to a specific hyperfine transition, which leads to a polarised population of the hyperfine level of the upper state as described in the previous section. The density matrix $\sigma_{A}=\sigma_{B}$ then follows by taking the partial trace of the density matrix for the sublevel $F_{e}$.

The number of independent parameters determining $G$ for a given value of the electronic angular momentum $J$ can be calculated from the symmetry relations $(3.3)-(3.6)$. This number amounts to $(\mathrm{J}+1)\left(4 \mathrm{~J}^{2}+5 \mathrm{~J}+3\right) / 3$ for integer $\mathrm{J}$-values, and to $(2 J+1)\left(8 J^{2}+14 J+9\right) / 12$ for half-integer $J$-values. This determines the number of independent measurements for a fuli determination of $G$. Both the polarisation and the beam direction of the exciting laser can be varied to produce a different value of the density matrices. A detailed analysis of the information extracted by measurement of the production rate of the final state with specific polarisation charac- 
teristics of the exciting light is given in a recent paper $/ 8 /$, where we focused on the case of associative ionisation. The same description is applicable to any inelastic process, provided that the total production rate of final-state particles can be measured.

A first measurement in our laboratory on polarisation effects on associative ionisation of excited sodium atoms was recently published $/ 5 /$. In the next section we will discuss some more extensive measurements.

\section{IV - MEASUREMENTS ON ASSOCIATIVE IONISATION}

We have performed measurements on the polarisation effect of associative ionisation of laser-excited sodium atoms, according to the reaction equation

$$
\mathrm{Na}\left(3 \mathrm{P}_{3 / 2}\right)+\mathrm{Na}\left(3 \mathrm{P}_{3 / 2}\right) \rightarrow \mathrm{Na}_{2}^{+}+\mathrm{e}
$$

Our earlier measurements were performed in a single atomic beam $/ 5 /$, where collisions are due to the spread in the atomic velocities in the beam. A remarkably strong polarisation effect was observed, when we detected the ion production rate as a function of the angle $\theta$ between the direction of the linear polarisation and the atomic-beam axis: the ion production was maximal for $\theta=0$, and minimal for $\theta=90^{\circ}$, with a ratio 1.5 . The detection operator $G$ contains 16 independent parameters for the $P_{3 / 2}$-state in the axially symmetric situation. If the reaction (4.1) is insensitive to the electron spin, then effectively $G$ acts only on the orbital angular momentum $L=1$ of the excited state, which would still yield 8 independent parameters. The ionisation signal as a function of $\theta$ has the form $/ 5,8 /$

$$
R(\theta)=R_{0}+R_{1} \cos 2 \theta+R_{2} \cos 4 \theta \quad,
$$

with $R_{0}, R_{1}$ and $R_{2}$ linear combinations of matrix elements of $G$. We adopted the simplifying assumption that the off-diagonal elements $<M_{A} \quad M_{B}|G| M_{A}^{\prime} M_{B}{ }^{\prime}>$ with $M_{A} \neq M_{A}^{\prime}, M_{B} \neq M_{B}^{\prime}$ are small compared to the diagonal ones, as a result of the integration in (3.2) over products of amplitudes with independent phases. The diagonal elements have the significance of $M$-dependent jonisation rates

$$
K\left(M_{A} M_{B}\right)=\left\langle M_{A} M_{B}|G| M_{A} M_{B}>,\right.
$$

which obeys the symmetry relations

$$
K\left(M_{A} M_{B}\right)=K\left(M_{B} M_{A}\right)=K\left(-M_{A}-M_{B}\right)
$$

Neglecting the off-diagonal terms gives rise to a direct determination of three independent combinations of rates from the measured values of $R_{0}, R_{1}$ and $R_{2}$. These relations for the $\mathrm{P}_{3 / 2}$ state are /5,8/

$$
\begin{aligned}
& K_{3}=R_{0}-\frac{7}{3} R_{1}+\frac{89}{9} R_{2} \\
& K_{2}=R_{0}-\frac{1}{3} R_{1}-\frac{79}{9} R_{2}
\end{aligned}
$$




$$
K_{1}=R_{0}+\frac{5}{3} R_{1}+\frac{41}{9} R_{2}
$$

with the abbreviations

$$
\begin{aligned}
& K_{3}=\frac{1}{2}\left[K\left(\frac{3}{2} \frac{3}{2}\right)+K\left(\frac{3}{2}-\frac{3}{2}\right)\right] \\
& K_{2}=\frac{1}{2}\left[K\left(\frac{3}{2} \frac{1}{2}\right)+K\left(\frac{3}{2}-\frac{1}{2}\right)\right] \\
& K_{1}=\frac{1}{2}\left[K\left(\frac{1}{2} \frac{1}{2}\right)+K\left(\frac{1}{2}-\frac{1}{2}\right)\right]
\end{aligned}
$$

In the absence of polarisation effects, we would have $k_{1}=K_{2}=K_{3}$, and $R_{1}$ and $R_{2}$ should have been zero. In fact, our first measurements indicated that $k_{1} / k_{3} \sim 1.6$, whereas $k_{2}$ was significantly smaller than either $k_{1}$ or $K_{3}$

\section{IVa - Counterrunning beams}

Reciently these measurements have been extended in two respects. First of all, the same measurement was performed with two counterrunning atomic beams, so that the axial symetry of the collision system was preserved. By subtracting the ionisation signal with only one of the two beams present, it is possible to isolate the rates $K$ for associative ionisation between two atoms, one from each of the two counterrunning beams. Hence the average relative velocity was much larger than in the case of a single beam. It turns out that the ratio $K_{1} / K_{3}$ becomes 2.0 , which is significantly larger than the value 1.6 in the case of a single beam. Details of the measurements will be published elsewhere. Here we emphasise the remarkable fact that the polarisation effects seem to get more pronounced when the relative velocity is increased. At first.sight this is surprising. One expects polarisation effects to be present when the various molecular states are unequaliy populated, and when the process of associative ionisation has unequal probabilities for the different quasi-molecular states of the collision complex. The non-uniform population distribution must be preserved during the collision in order to produce polarisation effects in the ion production, and this preservation of anisotropy is favoured by adiabatic behaviour of the molecular state. However, adiabaticity must be assumed to decrease for increasing relative velocity, whereas the polarisation effect is observed to increase. On the other hand, at higher relative velocity the electron spins can be expected to decouple from the internuclear axis, even when the orbital angular momentum of the two valence electrons is still coupled adiabatically to the axis, and the question arises whether the decoupling of the spin can explain the increase of the polarisation effect. This may be the case when the ionisation process occurs to a large extent by the molecular states which do have non-zero spin, i.e. the triplet states.

\section{IVb - Circular polarisation}

Another extension of the experiment was the use of circularly polarised laser light to excite the atoms. The 1 aser beam remained directed orthogonal to the atomic beam. In the stationary state, the atoms are in the pure state $|J M>=| \frac{3}{2} 3_{>}>$, when the laser beam is selected as quantisation axis. If we take the z-axis along the atomic beam, the steady state can be represented as the pure state

$$
\left[\left|\frac{3}{2} \frac{3}{2}>+\sqrt{ } 3\right| \frac{3}{2} \frac{1}{2}>+\sqrt{ } 3\left|\frac{3}{2}-\frac{1}{2}>+\right| \frac{3}{2}-\frac{3}{2}>\right] / \sqrt{ } 8
$$


since this is the eigenstate of $\mathrm{J}_{\mathrm{x}_{3}}$ with eigenvalue $\frac{3}{2}$. Hence the populations of the four magnetic substates with $M=\frac{x_{3}}{2}, \frac{1}{2},-\frac{1}{2}-\frac{3}{2}$ have the ratio's $1: 3: 3: 1$. When the off-diagonal terms of $G$ are ignored, we can predict the ion signal by using the rates $K_{1}, K_{2}$ and $K_{3}$ as determined from the case with linearly polarised laser light. Both in the case of a single atom beam and two counterrunning beams a significant deviation was observed: the measured rate with excitation with circularly polarised light was larger than the calculated value based on the observation with linearly polarised light. In fact, the ion signal with circularly polarised light was significantly larger than the maximum value (for $\theta=0^{\circ}$ ) of the ion signal with linearly polarised light, where the signals were normalised to the total observed excited-state population.

This result seems to indicate that our neglecting the off-diagonal terms of $G$ is not justified. This would mean that the coherences between magnetic substates contribute significantiy to the ion production. Note that the coherences between these states are maximal for excitation with circularly polarised light, since then the excited state is a pure state.

In the case of circular polarisation, the two electron spins in the initial state are both parallel to the laser beam, so that only molecular triplet terms are produced. The high ion production in this case suggests that the triplet states play an important part in the process of associative ionisation. This is not quite what one would expect, since the triplet states have an antisymmetric wave function of the two valence electrons, which tends to diminish the coulomb interaction needed for the reaction (4.1).

An explanation of these results in terms of the dynamics of the collision is not easy, since the potential curves of the system of two excited Na-atoms are not known in any detail. In fact, there are 12 quasimolecular terms ending up in the separated atoms $\mathrm{Na}(3 \mathrm{P})+\mathrm{Na}(3 \mathrm{P})$, with the designations $\mathrm{I}_{\Sigma_{\mathrm{g}}^{+}}^{+}, 1_{\Sigma_{\mathrm{g}}^{+}}^{+}, \mathrm{I}_{\Sigma_{u}}^{-},{ }_{1} \mathrm{I}_{\mathrm{g}},{ }_{\mathrm{I}_{u}},{ }_{1_{\Delta}}$, ${ }^{3} \Sigma_{U}^{+},{ }^{3} \Sigma_{U}^{+},{ }^{3} \Sigma_{g}^{-},{ }^{3} \Pi_{U},{ }^{3} \Pi_{g},{ }^{3} \Delta_{U}$. These terms produce 21 potential curves. The strong polarisation effects suggest that only a few of these participate in the ionisation process, probably since only a few cross with the ground-state potential curve of the molecular ion $\mathrm{Na}_{2}^{+}$.

The importance of the off-diagonal terms of $G$ can be derived from a complete experiment, using elliptically polarised light. The analys is of this experiment requires the carefui evaluation of the excited-state density matrix, along the lines of section II. This we intend to do in the near future. For the moment we may conclude from the observations that the polarisation effects increase when the velocity is increased from subthermal to thermal values, and that the off-diagonal terms of $G$ cannot be ignored. Since the same potential curves give rise to other energy-transfer processes, such as energy pooling, we can expect that similar conclusions hold for these cases.

\section{REFERENCES}

/1/ FANO U., MACEK J.H., Rev.Mod.Phys. 45 (1973) 553.

12) FANO U., RACAH G., Irreducible Tensorial Sets. (Academic, New York, 1959).

/3/ MACEK J., HERTEL I.V., J.PhyS. B7 (1974) 2173.

14/ DE VLIEGER G.J.N.E., HEIDEMAN H.G.M., VAN ECK J., NIENHUIS G., J.Phys. B15 (1982) L345.

15/ KIRCZ J.G., MORGENSTERN R., NIENHUIS G., Phys.Rev.Lett. 48 (1982) 610.

16/ COHEN-TANNOUDJI C., in: Frontiers in Laser Spectroscopy, R. Balian, S. Haroche, S. Liberman, eds. (North Holland, Amsterdam, 1977) 3.

17/ DUCLOY M., Phys.Rev. A8 (1973) 1844.

/8/ NIENHUIS G., Phys.Rev. A26 (1982) 3137. 